\title{
Relationship between oxygen cost and C-reactive protein response to marathon running in college recreational runners
}

This article was published in the following Dove Press journal: Open Access Journal of Sports Medicine

\author{
Fuminori Takayama ${ }^{1,2}$ \\ Atsushi Aoyagi ${ }^{3}$ \\ Keigo Takahashi ${ }^{2,3}$ \\ Yoshiharu Nabekura'
}

'Faculty of Health and Sport Sciences, University of Tsukuba, Tsukuba-shi, Ibaraki, Japan; ${ }^{2} J a p a n$ Society for the Promotion of Science, Chiyoda-ku, Tokyo, Japan; ${ }^{3}$ Graduate School of Comprehensive Human Sciences, University of Tsukuba, Tsukuba-shi, Ibaraki, Japan
Correspondence: Fuminori Takayama Faculty of Health and Sport Sciences, University of Tsukuba, I-I-I Tennodai Ibaraki, Ibaraki 305-8574, Japan

Tel +8I 298534959

Email takayama.fuminori.fw@u.tsukuba. ac.jp
Purpose: Individual variations in response of C-reactive protein (CRP) to acute strenuous exercise are less well known. The purpose of this study was to investigate the relationship between running economy and systemic inflammation following a marathon.

Materials and methods: Sixteen college recreational runners participated in this study. To measure maximal oxygen uptake and running economy, the treadmill running test was performed 1-2 weeks before the marathon race. Running economy was defined as oxygen cost $(\mathrm{mL} / \mathrm{kg} /$ $\mathrm{km}$ ) at submaximal running. CRP and muscle damage markers (creatine kinase and lactate dehydrogenase) were measured before and 1, 2, and 3 days after the race.

Results: All subjects completed the race in 4 hours 7 minutes 43 seconds \pm 44 minute 29 seconds [mean $\pm \mathrm{SD}$ ]. The marathon running significantly increased CRP and muscle damage markers. The levels of inflammation and muscle damage peaked after 1 day and remained high throughout the 3-day recovery period compared to that before the race. Spearman correlation analysis showed that the change in CRP level was significantly positively correlated with oxygen cost ( $r=0.619, P=0.011)$ but not maximal oxygen uptake. There was no significant relationship in responses between muscle damage markers and CRP.

Conclusion: These findings suggest that running economy is related to postmarathon race CRP response. Further study to clarify the cause of the relationship and clinical significance of transient increase in CRP is necessary.

Keywords: inflammation, oxygen consumption, C-reactive protein, muscle damage

\section{Introduction}

Running is one of the most popular forms of aerobic exercise. It is well known that habitual moderate aerobic exercise is effective in improving cardiovascular fitness. ${ }^{1}$ However, acute strenuous running, such as marathon running, can create acute physiological impacts. ${ }^{2-6}$ Biomarkers of muscle damage and inflammation have been measured as indicators of exercise stress. ${ }^{7}$ Kobayashi et al ${ }^{4}$ investigated creatine kinase (CK) and lactate dehydrogenase (LDH) to quantify muscle damage before and after a marathon race. In this report, post-race $\mathrm{CK}$ and $\mathrm{LDH}$ were significantly increased in comparison with pre-race value. C-reactive protein (CRP) is one of the markers of systemic inflammation following acute exercise. Kim et $\mathrm{al}^{2}$ investigated the CRP before and for 6 days after a marathon run and reported that it peaked 1 day after the race, returning to the prerace level on day 4 . The conclusion from these studies was that a marathon run has a negative impact on markers of muscle damage and systemic inflammation. 
Although the overall effects of marathon running on muscle damage and systemic inflammation have been investigated, individualization of factors that influence the magnitude of these responses has been less studied. It had been reported that differences in race time explained $32 \%$ of variability in changes in CRP after a marathon run. ${ }^{5}$ In addition, the most recent study indicated that high physical fitness was related to the attenuation of CRP elevation after an endurance cycling race. ${ }^{8}$ In this report, however, race time was used as a measure of physical fitness and no objective aerobic fitness markers were assessed. It is well known that endurance race performance is closely related to maximal oxygen uptake $\left(\mathrm{VO}_{2} \max \right)$ and running economy, which is defined as steady-state oxygen cost $\left(\mathrm{O}_{2}\right.$ cost $) .{ }^{9}$ In particular, the running economy is closely related to energy expenditure during the marathon race since the runner generates energy based on the oxygen consumption. ${ }^{10}$ In addition, one of the major energy sources is glycogen, as demonstrated by a significant reduction in glycogen concentration after a marathon run. ${ }^{11}$ Pedersen ${ }^{12}$ suggests that an acute increase in plasma IL-6, which stimulates CRP release via the hepatocyte, is dependent on muscle glycogen stores. Transcription of IL-6 is faster with low muscle glycogen content compared to high muscle glycogen content. ${ }^{12}$ Thus, it is reasonable to assume that CRP elevation post-race in runners with excellent running economies (ie, lower $\mathrm{O}_{2}$ cost) is minor because the energy expenditure is smaller. However, to our knowledge, the relationship between running economy and CRP response has not been taken into consideration to a significant extent thus far.

Kampus et $\mathrm{al}^{13}$ reported that acute elevation of the CRP after strenuous exercise may affect small artery elasticity, which is an important cardiovascular factor. Indeed, acute inflammation plays an important role in the development of atrial fibrosis and the progression to atrial fibrillation. ${ }^{14}$ Moreover, using two experimental groups consisting of a cherry juice (CJ) group and a placebo (PL) group, Dimitriou et $\mathrm{al}^{15}$ found that the CRP increase at 24 and 48 hours postmarathon was lower in the CJ group compared with the PL group. In the previous study, interestingly, no upper respiratory tract symptoms were reported in the CJ group, whereas $50 \%$ of runners in the PL group reported these symptoms after the race. Collectively, these previous studies indicate that excessive systemic inflammation after a marathon run is detrimental to health-related physiological functions. It is therefore important to understand factors related to individual variation in CRP response.

The purpose of the present study was to investigate the relationship between running economy and systemic inflammation following a marathon race. We hypothesized that running economy is related to post-race CRP response.

\section{Materials and methods Subjects}

Sixteen recreational runners (13 males and 3 females, aged $24.1 \pm 3$ years, height $170 \pm 7 \mathrm{~cm}$, and weight $61.6 \pm 10.2 \mathrm{~kg}$ $[$ mean $\pm \mathrm{SD}]$ ) participated in this study. The subjects were recruited through advertisement and word of mouth. All the subjects were free of injury and disease. All subjects had taken part in regular running training for the race. Training distance was $24 \pm 14 \mathrm{~km} /$ week and the longest training session was $33 \pm 19 \mathrm{~km}$ during the 3 months before the race.

Before participating, all subjects signed an informed consent, while ethical approval for the present study was granted by the Research Ethics Committee of the University of Tsukuba. This study was conducted according to the Declaration of Helsinki.

\section{Experimental design}

The present study was conducted before and after the 37th Tsukuba Marathon held in Ibaraki, Japan. This race was held on November 26, 2017 and was officially recognized by the Japan Association of Athletics Federations and a flat-road race course. At the start of the race, the temperature was $7.1^{\circ} \mathrm{C}$, the relative humidity $64 \%$, and wind speed $2.6 \mathrm{~m} / \mathrm{s}$.

To measure the aerobic fitness, a treadmill running test was performed 1-2 weeks before the race. Blood sampling was conducted 4 days before (PRE) and 1 (POST1), 2 (POST2), and 3 (POST3) days after the marathon race. The dependent variables measured were serum CK and serum LDH as muscle damage markers and serum CRP as indicator of systemic inflammation.

\section{Treadmill running test}

The treadmill running test was performed on a motorized treadmill (ORK-7000; Ohtake-Root Kogyo Co, Iwate, Japan) set at $1 \%$ gradient according to previous studies. ${ }^{9,16}$ This test consisted of a running economy test and a maximal incremental test. First, the subjects underwent a 5-minute running economy test at submaximal intensity that was set individually by the treadmill velocity at $85 \%$ of the velocity corresponding with the target velocity of the marathon race. Following a 5-minute recovery period after the running economy test, the maximal incremental test was performed. This test was initiated with the velocity at $8.4 \mathrm{~km} / \mathrm{h}$ and was increased by $0.6 \mathrm{~km} / \mathrm{h}$ per minute until volitional exhaustion. 
Expired gas analysis was performed on a breath-bybreath basis using the computerized standard open circuit technique by the metabolic gas analyzer (AE-310s; Minato Medical Science, Osaka, Japan). The data were analyzed by obtaining mean values at 20 -second intervals. Before the test, the metabolic system was calibrated using known gas concentrations and 2-L syringe according to the manufacturer's instructions. Heart rate (HR) was measured using a surface electrode chest strap (Polar H7 and M400; Polar Electro, Kempele, Finland).

The $\mathrm{O}_{2}$ cost was calculated based on the last 1-minute $\mathrm{VO}_{2}$ during the running economy test. $\mathrm{VO}_{2}$ max was defined as the attainment of at least two of the following four criteria: 1) a leveling-off $\mathrm{VO}_{2}$ despite an increase in the velocity; 2) peak respiratory exchange ratio (RER) $\geq 1.10,3$ ) peak $H R \geq 95 \%$ of the age-predicted HRmax, and 4) ratings of perceived exertion at the end of the test $\geq 19$. All subjects consumed a light meal at least 3 hours before the treadmill running test and during the 3 hours prior to the test, and water intake was allowed as needed.

\section{Blood sampling}

After overnight fasting, the blood sample ( $\sim 6 \mathrm{~mL}$ per sample) was drawn from an antecubital vein using a butterfly needle (Terumo, Tokyo, Japan) in a sitting position between 7:00 and 10:00 am. The collected blood was rapidly handed to the Tsukuba i-Laboratory LLP. After centrifugation at 3,000 rpm for 8 minutes, the serum was tested with an automatic clinical chemistry analyzer (LABOSPECT 008; Hitachi, Tokyo, Japan). CK and LDH were quantified by the Japan Society of Clinical Chemistry standardization method. The CRP was quantified using a latex coagulating nephelometry. The detection limit of CRP was $0.03 \mathrm{mg} / \mathrm{dL}$. Where activity was undetectable, the sample was assigned a value equivalent to the detection limit. The reference values were as follows: $\mathrm{CK}$, 63-257 U/L (male), 42-150 U/L (female); LDH, 106-211 $\mathrm{U} / \mathrm{L}$, and $\mathrm{CRP}<0.2 \mathrm{mg} / \mathrm{dL}$.

\section{Statistical analysis}

Data are expressed as means with SD. Friedman rank sums test and post hoc Wilcoxon-signed rank test were used for comparing between days. The relationships between selected variables were determined using Spearman correlation analysis. Because significant relationships were observed in each blood marker from POST1 to POST3, the POST1 values were taken as the representative value for the magnitude of damage. The Spearman correlation analysis was conducted based on delta difference between PRE and POST1. The statistical analyses were performed with SPSS Statistics 24 (IBM Japan, Tokyo, Japan).

\section{Results}

All subjects completed the race. The finishing time was $4 \mathrm{~h}$ $7 \mathrm{~min} 43 \mathrm{~s} \pm 44 \min 29 \mathrm{~s}$ (range: $2 \mathrm{~h} 27 \mathrm{~min} 16 \mathrm{~s}$ to $5 \mathrm{~h} 0 \mathrm{~min}$ $11 \mathrm{~s})$. The average velocity during the race was $10.6 \pm 2.4 \mathrm{~km} / \mathrm{h}$.

\section{Aerobic fitness}

The subjects had the following characteristics: $\mathrm{VO}_{2} \max$ of $57.4 \pm 7.1 \mathrm{~mL} / \mathrm{kg} / \mathrm{min}$ (range: $47.9-75.5 \mathrm{~mL} / \mathrm{kg} / \mathrm{min}$ ), $\mathrm{O}_{2}$ cost of $225.1 \pm 21.3 \mathrm{~mL} / \mathrm{kg} / \mathrm{km}$ (range: $193.6-264.8 \mathrm{~mL} / \mathrm{kg} / \mathrm{km}$ ). In the running economy test, the RER of all subjects was $<1.0$.

\section{Changes in markers of muscle damage and inflammation}

Figures 1 and 2 show the changes in muscle damage markers before and after the marathon race. There were significant effects for each subsequent day following the race, and post hoc analyses showed that the peak was achieved on POST1 and remained high throughout the 3-day recovery period compared to PRE.

Figure 3 shows the change in CRP before and after a marathon run. The CRP level peaked on POST1 and remained high throughout the 3-day recovery period compared to PRE. All subjects had at least one above reference value at 3 days after the race.

\section{Relationship between the variables}

The coefficient of variation at POST1 was $73.2 \%$ for CK, $58.3 \%$ for $\mathrm{LDH}$, and $71.3 \%$ for CRP, showing remarkable individual variations. Table 1 shows the relationships between the variables. $\mathrm{VO}_{2}$ max was not related to $\mathrm{CK}, \mathrm{LDH}$, and CRP at POST1. There was a significant positive correlation in $\mathrm{O}_{2}$ cost with CRP elevation at POST1 $(r=0.619, P=0.011)$ (Figure 4). $\mathrm{O}_{2}$ cost was not related to $\mathrm{CK}$ and $\mathrm{LDH}$. When excluding female subjects, the significant relationship was unchanged (shown in parentheses).

A significant relationship was observed between CK and LDH. However, CRP was not related to CK and LDH. There was a significant positive correlation between race time ( $\mathrm{min})$ and CRP elevation at POST1 $(r=0.647, P=0.007)$ and a significant negative correlation to $\mathrm{VO}_{2} \max (r=-0.609$, $P=0.012$ ).

\section{Discussion}

The purpose of the present study was to investigate the relationship between running economy and systemic inflamma- 


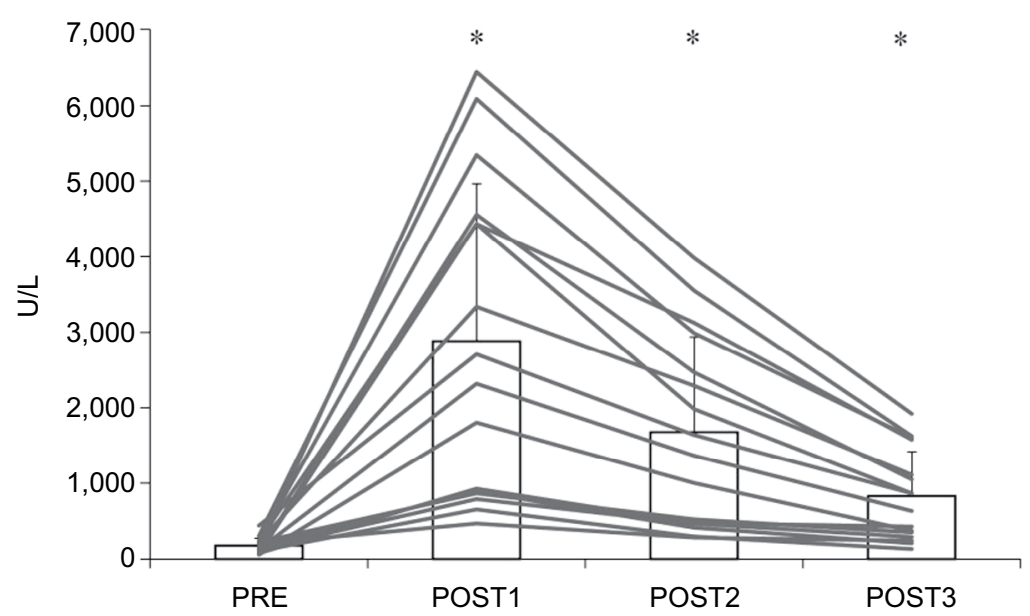

Figure I Serum creatine kinase before and after a marathon run $(n=16)$.

Notes: ${ }^{*} P<0.05$ - significant difference compared to PRE. Gray lines indicate individual subject results and bar graph indicates the group mean with SD values.

Abbreviations: PRE, blood sampling done 4 days before the marathon race; POSTI, blood sampling done I day after the marathon race; POST2, blood sampling done I day after the marathon race; POST3, blood sampling done 3 days after the marathon race.

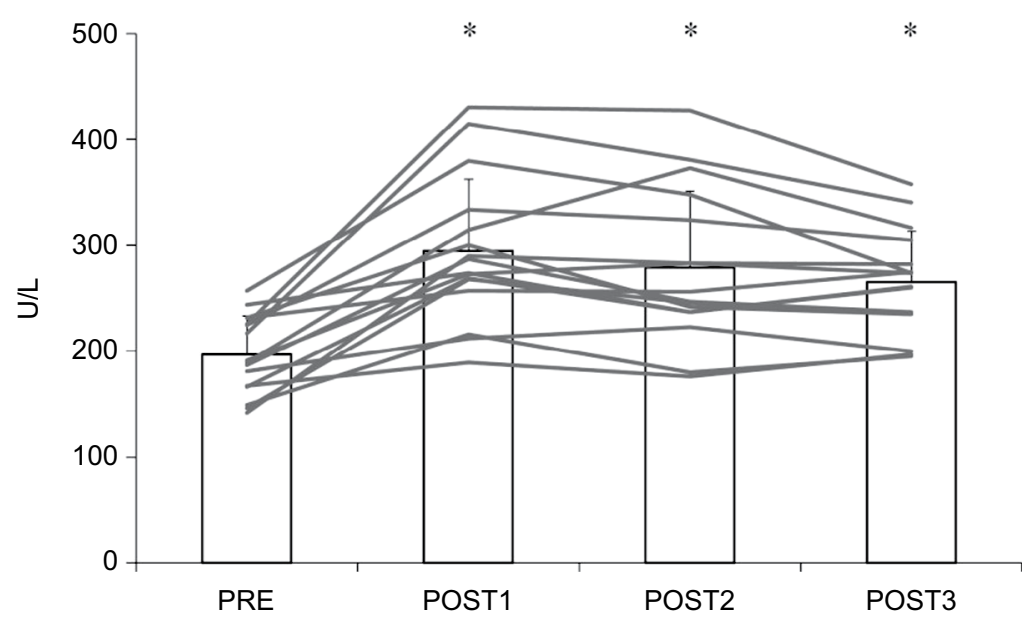

Figure 2 Serum lactate dehydrogenase before and after a marathon run $(n=16)$

Notes: $* P<0.05$ - significant difference compared to PRE. Gray lines indicate individual subject results and bar graph indicates the group mean with SD values.

Abbreviations: PRE, blood sampling done 4 days before the marathon race; POSTI, blood sampling done I day after the marathon race; POST2, blood sampling done I day after the marathon race; POST3, blood sampling done 3 days after the marathon race.

tion after a marathon race. The most important finding was that $\mathrm{O}_{2}$ cost is related to post-race CRP response in college recreational runners. This finding suggests that systemic inflammation is minor in runners with superior running economies.

$\mathrm{VO}_{2}$ max and running economy were recognized as the main physiological factors determining marathon running performance. Of the two factors, $\mathrm{VO}_{2} \max$ had the larger impact in heterogeneous performance runners, ${ }^{9}$ supported by the present result showing the relationship between aerobic fitness and race time (Table 1). Several studies have investi- gated the relationship between $\mathrm{VO}_{2}$ max and CRP response after acute exercise. ${ }^{3,17} \mathrm{Kim}$ et $\mathrm{al}^{3}$ investigated the relationship between $\mathrm{VO}_{2}$ max and CRP response immediately after a marathon race and reported no relationship between them $(r=-0.03)$. A previous study by Plaisance et $a{ }^{17}$ showed that response of systemic inflammation after acute aerobic exercise was no different between individuals of at least average peak oxygen uptake levels. In the present study, although the value of $\mathrm{VO}_{2} \max$ is heterogeneous, all subjects achieved a value greater than the reference value for a population of the same age and sex. ${ }^{18}$ Thus, higher $\mathrm{VO}_{2} \max$ does not appear 


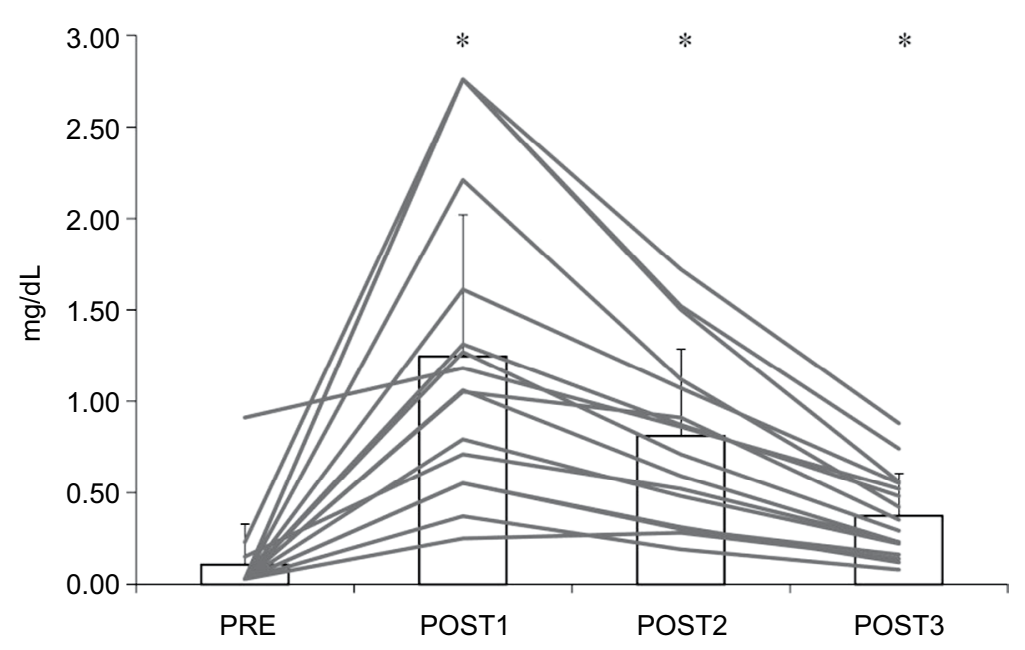

Figure 3 Serum C-reactive protein before and after a marathon run $(n=16)$.

Notes: ${ }^{*} P<0.05$ - significant difference compared to PRE. Gray lines indicate individual subject results and bar graph indicates the group mean with SD values.

Abbreviations: PRE, blood sampling done 4 days before the marathon race; POSTI, blood sampling done I day after the marathon race; POST2, blood sampling done I day after the marathon race; POST3, blood sampling done 3 days after the marathon race.

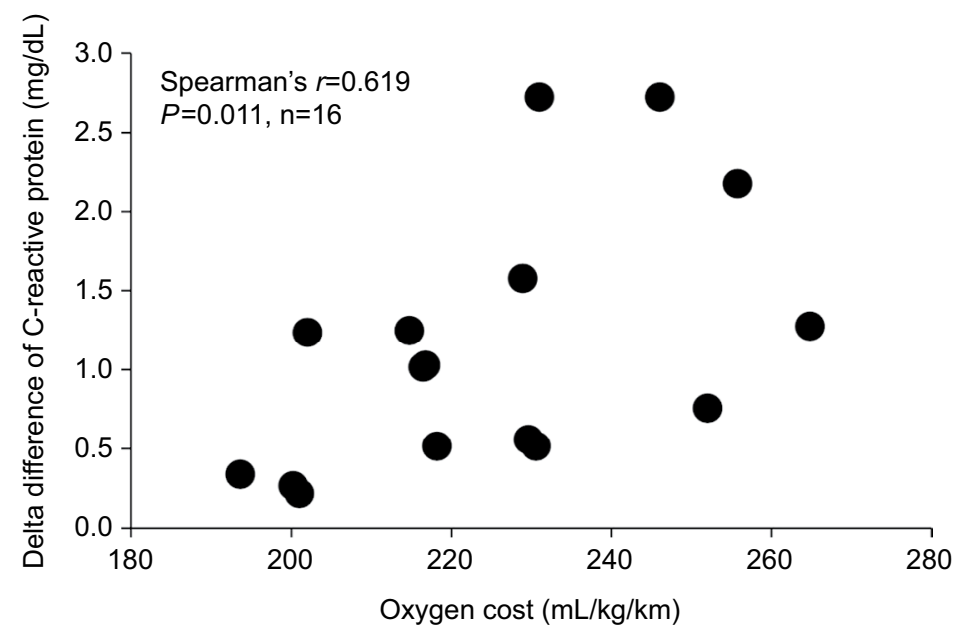

Figure 4 Relationship between oxygen cost and delta difference of C-reactive protein $(n=16)$.

Notes: Delta difference is calculated between PRE and POSTI.

Abbreviations: PRE, blood sampling done 4 days before the marathon race; POSTI, blood sampling done I day after the marathon race.

Table I Spearman correlation analysis of aerobic fitness, race time, and blood sampling I day after a marathon run

\begin{tabular}{|l|l|l|l|l|l|}
\hline Variables & VO $_{2} \max$ & $\mathbf{O}_{2}$ cost & Race time & CK & LDH \\
\hline VO $_{2}$ max & - & & & & \\
$\mathrm{O}_{2}$ cost & $0.096(-0.135)$ & - & & & \\
Race time & $-0.609^{*}\left(-0.746^{*}\right)$ & $0.368(0.407)$ & - & & \\
$\mathrm{CK}$ & $-0.147(-0.281)$ & $0.209(0.291)$ & $0.374(0.456)$ & - & \\
LDH & $-0.218(-0.253)$ & $0.091(0.214)$ & $0.450\left(0.555^{*}\right)$ & $0.844^{*}\left(0.79 I^{*}\right)$ & - \\
CRP & $-0.066(-0.450)$ & $0.619^{*}\left(0.567^{*}\right)$ & $0.647^{*}\left(0.725^{*}\right)$ & $0.427(0.399)$ & $0.255(0.278)$ \\
\hline
\end{tabular}

Notes: $* P<0.05$, significant correlation. Parentheses indicate excluding females.

Abbreviations: $\mathrm{VO}_{2} \max$, maximal oxygen uptake; $\mathrm{O}_{2}$ cost, oxygen cost; $\mathrm{CK}$, creatine kinase; $\mathrm{LDH}$, lactate dehydrogenase; CRP, C-reactive protein.

to be related to superior resistance to systemic inflammation after a marathon race in young recreational runners.

Unlike other inflammatory markers, CRP levels peak 24 hours after a marathon race. Thus, since we could not conduct blood sampling immediately after the race, $\mathrm{CRP}$ was selected to assess the systemic inflammation. A significant positive relationship was observed between $\mathrm{O}_{2}$ cost and CRP response after a marathon race, suggesting that systemic inflammation 
in runners with superior running economies was minor. This is an important finding because acute excessive systemic inflammation has detrimental effects on health-related physiological functions as discussed in the introduction. The detailed mechanism is unknown but it may be that energy expenditure is different during a marathon race. Since the runners generate energy based on the amount of oxygen consumed during submaximal running, $\mathrm{O}_{2}$ cost can estimate energy expenditure ( $1 \mathrm{~L}_{\text {of }} \mathrm{O}_{2}$ has $\sim 5 \mathrm{kcal}$ ). Assuming that the running economy was similar among the participants during the race, the difference in energy expenditure was calculated to reach $37 \%$ in the present subjects. Therefore, it is possible that differences in energy expenditure affected the extent of systemic inflammation following the race.

It is known that causes of increased CRP levels include repeated hypoxic stress. ${ }^{19,20}$ Guven et $\mathrm{al}^{19}$ reported that the increased CRP levels were observed in subjects with obstructive sleep apnea and were related to oxygen desaturation. A high $\mathrm{O}_{2}$ cost means that oxygen consumption by the muscles is high. Therefore, the significant relationship between $\mathrm{O}_{2}$ cost and CRP response after a marathon race is likely in part due to oxygen desaturation during the race. However, to our knowledge, no study has compared $\mathrm{O}_{2}$ cost and CRP response. Furthermore, oxygen saturation is closely affected by relative intensity (ie, $\left.\% \mathrm{VO}_{2} \max \right) .{ }^{21}$ In the present study, we did not measure HR or oxygen saturation during the race. Thus, there is a need to further investigate the cause of the significant relationship found between $\mathrm{O}_{2}$ cost and CRP response.

A significant positive relationship was observed between race time and CRP response, showing that longer race time increases the CRP response. This finding is in agreement with the result of several previous studies for marathon running ${ }^{5}$ or an endurance cycling race. ${ }^{8}$ In the present study, interestingly, even though no significant relationship was observed between $\mathrm{O}_{2}$ cost and race time, the $\mathrm{O}_{2}$ cost was significantly related to $\mathrm{CRP}$ response. This result indicates that running economy may be related to systemic inflammation after marathon running, independently of race time.

Several previous studies indicated a similar response of both CRP and CK after acute exercise. ${ }^{22,23}$ For example, a previous investigation in an Ironman triathlon showed a significant relationship between responses of CK and CRP 1 day after the race. ${ }^{23}$ In contrast, no significant relationships were observed between muscle damage markers and CRP response in the present study. In a well-controlled laboratory study, Croisier et $\mathrm{al}^{24}$ measured serum myoglobin as a marker of muscle damage, as well as IL-6 after two damaging eccentric exercise sessions, separated by a period of 3 weeks during which the subjects underwent five training sessions. After training, serum myoglobin was significantly lower than that measured before training, suggesting that these training sessions conferred a protective effect against muscle damage. However, the response of IL-6 was no different before and after training. Based on this result, they concluded that there was no causal relationship between exercise-induced muscle damage and post-exercise elevation in IL-6. Taking all these findings into account, the influence of muscle damage on systemic inflammation after marathon running appears to be minor.

A significant relationship was observed between race time and LDH response when excluding females, suggesting longer race time induces more severe muscle damage. It is well known that exercise-induced muscle damage is attenuated in well-trained subjects by the repeated bout effect. ${ }^{25}$ In brief, a fast runner is well trained and has a strong protective effect against muscle damage. Indeed, a previous study showed that muscle damage was more severe in the novice runner group (average race velocity: $9.5 \mathrm{~km} / \mathrm{h}$ ) compared to an experienced runner group (average race speed $13.8 \mathrm{~km} / \mathrm{h}$ ) after a 56-km ultramarathon race. ${ }^{26}$ Therefore, the significant relationship between race time and LDH may be caused by the differences in protective effects against muscle damage in the individual runners.

In the present study, there were significant differences in markers of muscle damage and inflammation between PRE and POST3. Moreover, all subjects had at least one above reference value at 3 days after the race. These results indicate that, in college recreational runners, a 3-day recovery period does not seem to be long enough for recovery following a marathon race. Retraining in a state of insufficient recovery could lead to further increase in $\mathrm{CK}$ after a marathon. ${ }^{27}$ Meanwhile, it has been reported that physiological capacity recovered within 7 days of a marathon race. ${ }^{16}$ Therefore, we encourage recreational unners to take a passive rest for at least 3 days after the race, but recommend that they can resume training 1 week later.

The present study was a field study, and thus we acknowledge several limitations. The first limitation is that evaluation of running economy was based on the result of the treadmill running test and not on measuring muscle glycogen stores. Therefore, the causal relationship between running economy and glycogen stores after the race is unknown. Second, we did not control food intake during the experimental period. Third, the present study included both males and females. However, 
the significant relationship between $\mathrm{O}_{2}$ cost and CRP response was unchanged when excluding females. Finally, the present study has a cross-sectional design and relatively small sample size. Further studies should be conducted to clarify causal relationships between running economy and CRP response after marathon running.

\section{Conclusion}

The present study confirmed the hypothesis that $\mathrm{O}_{2}$ cost is related to postmarathon race CRP response. This finding suggests that systemic inflammation is minor in the runners with superior running economies. Further study to clarify the cause of the relationship and clinical significance of transient increase in CRP is necessary.

\section{Acknowledgments}

This work was supported by Grant-in-Aid for JSPS Research Fellow (No. 17J01363). The authors would like to thank Dr N Mukai for assistance in blood sample collection. We would like to thank Editage (www.editage.jp) for English language editing. Part of this study was presented in Oral Presentation format at 23rd Annual Congress of the European College of Sport Science, Dublin, Ireland, 4-7 July 2018 (Session-ID: OP-PM80).

\section{Disclosure}

The authors report no conflicts of interest in this work.

\section{References}

1. Plaisance EP, Grandjean PW. Physical activity and high-sensitivity C-reactive protein. Sports Med. 2006;36(5):443-458.

2. Kim HJ, Lee YH, Kim CK. Changes in serum cartilage oligomeric matrix protein (COMP), plasma CPK and plasma hs-CRP in relation to running distance in a marathon $(42.195 \mathrm{~km})$ and an ultra-marathon (200 km) race. Eur J Appl Physiol. 2009;105(5):765-770.

3. Kim YJ, Ahn JK, Shin KA, Kim CH, Lee YH, Park KM. Correlation of cardiac markers and biomarkers with blood pressure of middle-aged marathon runners. J Clin Hypertens. 2015;17(11):868-873.

4. Kobayashi Y, Takeuchi T, Hosoi T, Yoshizaki H, Loeppky JA. Effect of a marathon run on serum lipoproteins, creatine kinase, and lactate dehydrogenase in recreational runners. Res $Q$ Exerc Sport. 2005;76(4):450-455.

5. Mündermann A, Geurts J, Hügle T, et al. Marathon performance but not BMI affects post-marathon pro-inflammatory and cartilage biomarkers. J Sports Sci. 2017;35(7):711-718.

6. Wilhelm M, Zueger T, De Marchi S, et al. Inflammation and atrial remodeling after a mountain marathon. Scand J Med Sci Sports. 2014;24(3):519-525

7. Bessa AL, Oliveira VN, Agostini GG, et al. Exercise intensity and recovery: biomarkers of injury, inflammation, and oxidative Stress. $J$ Strength Cond Res. 2016;30(2):311-319.
8. Kleiven $\varnothing$, Bjørkavoll-Bergseth M, Melberg T, et al. High physical fitness is associated with reduction in basal- and exercise-induced inflammation. Scand J Med Sci Sports. 2018;28(1):172-179.

9. Takayama F, Aoyagi A, Nabekura Y. Relationship between classic physiological variables and running performance in recreational runners. Int J Sport Health Sci. 2018;16:33-40.

10. Rapoport BI. Metabolic factors limiting performance in marathon runners. PLoS Comput Biol. 2010;6(10):e1000960.

11. Asp S, Daugaard JR, Rohde T, Adamo K, Graham T. Muscle glycogen accumulation after a marathon: roles of fiber type and pro- and macroglycogen. J Appl Physiol. 1999;86(2):474-478.

12. Pedersen BK. Muscular interleukin- 6 and its role as an energy sensor. Med Sci Sports Exerc. 2012;44(3):392-396.

13. Kampus P, Kals J, Unt E, et al. Association between arterial elasticity, $\mathrm{C}$-reactive protein and maximal oxygen consumption in well-trained cadets during three days extreme physical load: a pilot study. Physiol Meas. 2008;29(4):429-437.

14. Swanson DR. Atrial fibrillation in athletes: implicit literature-based connections suggest that overtraining and subsequent inflammation may be a contributory mechanism. Med Hypotheses. 2006;66(6):1085-1092.

15. Dimitriou L, Hill JA, Jehnali A, et al. Influence of a montmorency cherry juice blend on indices of exercise-induced stress and upper respiratory tract symptoms following marathon running: a pilot investigation. J Int Soc Sports Nutr. 2015;12:22.

16. Takayama F, Aoyagi A, Shimazu W, Nabekura Y. Effects of marathon running on aerobic fitness and performance in recreational runners one week after a race. $J$ Sports Med. 2017;2017:1-6.

17. Plaisance EP, Taylor JK, Alhassan S, Abebe A, Mestek ML, Grandjean PW. Cardiovascular fitness and vascular inflammatory markers after acute aerobic exercise. Int J Sport Nutr Exerc Metab. 2007;17(2):152-162.

18. Ministry of Health Labour and Welfare of Japan [homepage on the Internet]. Physical activity reference for health promotion; 2013. Available from: https://www.mhlw.go.jp/stf/houdou/2r9852000002xpleatt/2r9852000002xpqt.pdf. Accessed July 29, 2018.

19. Guven SF, Turkkani MH, Ciftci B, Ciftci TU, Erdogan Y. The relationship between high-sensitivity C-reactive protein levels and the severity of obstructive sleep apnea. Sleep Breath. 2012;16(1):217-221.

20. Hartmann G, Tschöp M, Fischer R, et al. High altitude increases circulating interleukin-6, interleukin-1 receptor antagonist and C-reactive protein. Cytokine. 2000;12(3):246-252.

21. Peltonen JE, Leppävuori AP, Kyrö KP, Mäkelä P, Rusko HK. Arterial haemoglobin oxygen saturation is affected by $\mathrm{F}(\mathrm{I}) \mathrm{O} 2$ at submaximal running velocities in elite athletes. Scand J Med Sci Sports. 1999;9(5):265-271.

22. Kim HJ, Lee YH, Kim CK. Biomarkers of muscle and cartilage damage and inflammation during a $200 \mathrm{~km}$ run. Eur J Appl Physiol. 2007;99(4):443-447.

23. Neubauer O, König D, Wagner KH. Recovery after an Ironman triathlon: sustained inflammatory responses and muscular stress. Eur J Appl Physiol. 2008;104(3):417-426.

24. Croisier JL, Camus G, Venneman I, et al. Effects of training on exerciseinduced muscle damage and interleukin 6 production. Muscle Nerve. 1999;22(2):208-212.

25. Mchugh MP, Connolly DA, Eston RG, Gleim GW. Exercise-induced muscle damage and potential mechanisms for the repeated bout effect. Sports Med. 1999;27(3):157-170.

26. Noakes TD, Carter JW. The responses of plasma biochemical parameters to a $56-\mathrm{km}$ race in novice and experienced ultra-marathon runners. Eur J Appl Physiol Occup Physiol. 1982;49(2):179-186.

27. Maresh CM, Allison TG, Noble BJ, Drash A, Kraemer WJ. Substrate and hormone responses to exercise following a marathon run. Int $J$ Sports Med. 1989;10(2):101-106. 


\section{Publish your work in this journal}

The Open Access Journal of Sports Medicine is an international, peer-reviewed, open access journal publishing original research, reports, reviews and commentaries on all areas of sports medicine. The journal is included on PubMed. The manuscript management system is completely online and includes a very quick and fair

Submit your manuscript here: http://www.dovepress.com/open-access-journal-of-sports-medicine-journal

peer-review system. Visit http://www.dovepress.com/testimonials.php to read real quotes from published authors. 